\title{
GESTION PUBLIQUE ET GESTION MARCHANDE DU TRANSPORT FERROVIAIRE
}

\author{
Christian Barrère \\ professeur de Sciences économiques à l'Université de Reims Champagne Ardenne, laboratoire \\ Ceras-EDJ (Economie-Droit-Justice).

\section{Le modèle français de gestion publique du transport ferroviaire et sa crise}

\section{Le modèle des années 60}

Le service public du transport prend des formes qui découlent d'une conception précise du service public, la conception française du service public. Celle-ci donne à l'Etat une place à côté du marché mais aussi au-dessus du marché. Elle peut se définir comme une conception politique "républicaine" parce qu'elle donne au service public un fondement politique dérivé de l'idéologie républicaine. Le service public correspond en premier lieu à la volonté d'assurer, hors logique marchande, la fourniture des biens et services de base permettant l'intégration des individus dans la société en tant que citoyens et quel que soit leur statut économique marchand. Le principe d'intégration républicaine est la traduction de la socialisation politique. Le contenu des services publics n'est pas d'abord affaire de conditions technologiques mais de projet de société. Ce fondement du service public est de type distributif.

Un second fondement républicain du service public s'y ajoute. II s'inscrit dans une démarche volontariste qui exprime le refus de soumettre la société à la seule logique du marché et à faire du développement social la résultante non maîtrisée du développement économique du marché. L'Etat des citoyens est responsable de la dynamique sociale. Celle-ci ne doit pas résulter de la dynamique du marché mais d'un choix politique effectué par des individus égaux, selon le principe un homme/une voix et non, comme dans la dynamique marchande, selon l'inégalité des pouvoirs économiques. L'Etat définit des orientations de l'avenir et est un moyen de la maîtrise par la société de son propre développement. II est donc compétent sur les fins et les moyens du développement. A côté de ses fonctions régaliennes, qui expriment au fond l'égalité des hommes en tant que citoyens, I'Etat exprime des choix de société et est l'organe de leur concrétisation. II se donne pour cela des moyens, les deux principaux étant la régulation publique (dans laquelle prend place le service public) et la régulation marchande. Un tel fondement dépasse largement l'aspect distributif précédent. Le service public est public en ce qu'il participe de missions d'intérêt général. Par principe, l'Etat est au-dessus de la régulation marchande, à laquelle il n'est pas soumis mais qu'il utilise. La République passe avant le marché.

Cette conception est profondément ancrée dans la nation parce que c'est la République qui, en France, marque le passage à la modernité et en définit les structures de base. L'ancrage résulte aussi de ce que le fondement politique républicain a servi de creuset à la convergence de grands courants de pensée qui ont, par la suite, profondément marqué la société française et défini sa culture. II s'est en effet progressivement enrichi d'un fondement économique et social lorsque la tradition républicaine du service public a rencontré plusieurs autres traditions : la tradition keynésienne de l'économie mixte, la tradition sociale caritative du christianisme social (la loi et l'Etat défenseurs des pauvres), la tradition solidariste de la social-démocratie (I'Etat défenseur des faibles et réducteur d'inégalité), enfin la tradition scientifico-technocratique française des administrateurs et ingénieurs des corps d'Etat (l'Etat organisateur du progrès économique et scientifique).

Dans le domaine du transport, la forme prise par le principe d'intégration républicaine est le droit au transport. Ce choix socio-politique part de l'idée selon laquelle le besoin de transport est un besoin social dont la satisfaction doit être garantie à chaque membre de la communauté ; la LOTI de 1982 affirme explicitement que les citoyens disposent d'un droit au transport et que l'Etat, organe de la souveraineté nationale, doit prendre les moyens de garantir l'exercice de ce droit. Et 
c'est par rapport à ce principe que la doctrine française du service public a défini les caractères que doit remplir la fourniture du service public (continuité, égalité d'accès et de traitement, adaptation aux besoins). Les moyens d'assurer le droit au transport différent selon les modes de transport, étant donné qu'il peut y avoir des missions de service public confiées à des opérateurs privés. Les formes publiques sont cependant privilégiées parce que la politique des services publics s'accompagne d'une politique d'interventionnisme étatique. La reconstruction d'aprèsguerre s'est faite sous l'égide de l'Etat et ce dernier a consolidé la croissance, en orientant et soutenant le développement économique. La volonté d'une maîtrise publique du devenir implique des moyens de peser sur cet avenir et donc des centres d'accumulation de capital échappant à la stricte logique de la rentabilité financière à court terme. Dans le domaine du transport ferroviaire, cela a conduit à une gestion à dominante publique d'un service de transport défini en tant que tel comme service public.

La gestion publique du transport s'est progressivement donné des objectifs complémentaires d'économie de coûts par la gestion centralisée d'un réseau permettant de développer économies d'échelle et d'envergure, puis des objectifs d'aménagement du territoire et de protection de l'environnement, qui ont conduit à substituer un critère d'efficacité économique et sociale à celui de rentabilité financière. Elle s'est incarnée dans un modèle, celui de la grande entreprise nationale de transport, développé après guerre, qui a pris sa forme classique dans les années 50 et 60 pour entrer en crise, après diverses adaptations (dont l'adoption du statut d'EPIC') dans les années 80 et 90.

Le modèle des années 60 définit de fait le service public du transport ferroviaire comme un service performant pour les usagers, délivré à prix raisonnable, dont la rentabilité n'est pas l'objectif, avec péréquation géographique, articulé sur une politique industrielle et une politique d'aménagement du territoire, donc soucieux du développement de long terme.

\section{Limites et crise du modèle}

Le modèle des années 60 du transport ferroviaire a beaucoup apporté. II a participé à la croissance des années 50,60 et 70 . Il est aujourd'hui dépassé du fait de son incapacité à gérer le service de transport en tenant compte à la fois de sa dimension de service public et de ses autres dimensions. II éclate sous la pression apparente de trois types de mutations, dans une conjoncture longue particulière, l'ensemble de ces données posant en termes nouveaux les problèmes de fond de la politique du transport :

1 - le recouvrement total entre service public et entreprise publique est remis en cause parce que le modèle du secteur clé à dynamique publique est aujourd'hui dépassé. Avec l'ouverture des marchés européens à une concurrence exacerbée, le fractionnement et la segmentation des marchés, l'instabilité technologique, les règles du jeu changent. Le modèle quasi-militaire de la mobilisation de ressources sur un programme donné avec définition d'objectifs à moyen terme, sur le principe du colbertisme, ne convient plus à une gestion complexe en univers instable qui demande adaptation rapide, souplesse, négociation avec les partenaires-concurrents. En outre, il s'avère coûteux en développant diverses inefficacités, du fait d'asymétries d'information qui rendent le contrôle étatique peu performant et des biais traditionnels de la gestion politique du contrôle.

2 - les mutations technologiques des produits et procédés de production modifient les frontières entre secteurs et introduisent dans le périmètre du service public des productions qui n'y correspondent plus et n'ont aucune raison d'être gérées selon sa logique. Ainsi la SNCF metelle en place des activités purement commerciales en développant, à partir de son acquit dans l'interconnexion de réseau, ses opérations dans le secteur des télécommunications. La mondialisation joue de la même façon : la SNCF investit à l'étranger (dans le transport, l'hôtellerie ou les télécommunications) sans que cela corresponde à une nécessité pour la fourniture d'un service public national.

1 Le statut d'EPIC, établissement public à caractère industriel et commercial, est une tentative de prendre en compte, au sein de la régulation publique, la dimension marchande de l'activité des entreprises publiques. 
3 - le contenu de la demande adressée au service public change 2 . Les demandes évoluent et se différencient et la satisfaction d'un service standard n'est plus de mise. Alors qu'elle offrait, dans les années 50 et 60 , un service de base relativement homogène (distinguant seulement entre transport voyageur et transport marchandise), l'entreprise publique, dans une économie de services, rencontre désormais et des usagers et des clients, diversifie son offre voyageur et son offre marchandise et doit les gérer de plus en plus selon une logique de clientèle et non une logique administrative. Une différenciation verticale traduit les exigences diversifiées des utilisateurs en matière de qualité du service et se mélange à une différenciation horizontale liée à la diversité des besoins de transport.

Ces mutations interviennent dans un contexte de globalisation hyper-concurrentielle des marchés et d'intégration des systèmes de transport. Les deux problèmes traditionnels du transport, celui de l'unité entre les formes marchandes et les formes non marchandes de sa gestion, celui de l'unité intermodale du transport, se présentent alors en termes nouveaux :

- le modèle de gestion du transport était fondé sur une conception de l'économie mixte divisant la sphère économique en deux sous-ensembles relativement hermétiques : un sousensemble non marchand et public, un sous-ensemble marchand et privé. Le domaine du transport ferroviaire participait du premier et était donc régi quasi-exclusivement par une régulation non marchande. Puisque chacun a droit au service public indépendamment de son statut économique le service de transport aurait pu être gratuit, une redistribution s'opérant alors par le mécanisme prélèvement fiscal/fourniture de service public.

- la prise en compte d'un risque de gaspillage a conduit à imposer un tarif uniforme, mais la réalité des diversités de goûts et de coûts modifie les choses. Une gestion économique plus complexe oblige désormais à considérer de façon plus fine, plus détaillée, les problèmes de coût du service public. Si le service public doit conquérir son autonomie par rapport à la logique marchande, il ne peut être indifférent à toute considération de coût, a fortiori en période de crise financière des Etats. Dès que le service public devient complexe et coûteux le bilan coûtavantage est nécessaire et, puisque le service sera payé de toutes façons par les contribuables, le principe d'économie a un sens.

- de même, du fait de la différenciation des consommations, il est utile que le service fourni corresponde à ce qui est demandé sans offrir de surproduction coûteuse, quitte à subventionner une partie des demandes pour permettre l'accès de tous. La régulation non marchande à fondement politique doit s'articuler à une prise en compte de l'économique et du marchand, à la fois dans la définition de ce qui sera service public et dans celle des modes de gestion. Est-il possible de faire circuler sur tout le territoire des trains proposant les mêmes services, la même vitesse, la même fréquence, et cela au même tarif ? Le service public doit-il inclure la totalité des segments ainsi découpés ? Doit-il se limiter à un service basique, sorte de service homogène universel et voir les prestations adaptatives fournies, sur une base commerciale, par l'entreprise publique et/ou des firmes privées?

Faute d'avoir posé ces questions explicitement, le système actuel mélange sans principe données non marchandes et marchandes. Celles-ci n'ont guère de signification, le meilleur exemple en étant le niveau de déficit financier, amalgame sans signification ni politique ni économique.

- la logique marchande s'impose directement par le biais de la concurrence des autres modes de transport, eux mêmes régis prioritairement par une logique marchande. Le cas du fret SNCF est significatif : censé obéir à une logique de service public, sa politique est, de facto, gouvernée par la nécessité concurrentielle de s'aligner sur les tarifs du fret routier. On aboutit ainsi à une gestion, en principe non marchande et, en réalité, de plus en plus gouvernée par le marché, sans que cela soit explicitement assumé et qu'une gestion cohérente en découle (par exemple en matière de définition et de charge des investissements).

La politique du ferroviaire subit alors les conséquences d'une politique de la route qui obéit à d'autres principes, et dans sa gestion publique (politique des infrastructures routières) et dans sa

2 Elles se traduisent notamment par des dispositions à payer différentes pour les caractéristiques de rapidité et d'adaptabilité du transport. Les demandes, loin de se cantonner à un service de base universel et homogène, évoluent et se particularisent. Les demandes de transport des personnes du troisième âge ne sont pas les mêmes que celles des institutions scolaires, des hommes d'affaires,...et cette réalité est encore plus accusée en matière de fret. 
gestion privée (stratégies des chargeurs et des transporteurs, stratégies des constructeurs automobiles et comportements des ménages) sans qu'une politique globale des transports n'ait été définie et ne soit mise en œuvre. Les résultats - au rang desquels la crise financière de la SNCF, le déclin du fret ferroviaire, l'hégémonie du transport routier de marchandises, la dégradation accélérée de l'environnement - en sont d'autant plus catastrophiques que la concurrence rail-route, dans un contexte européen de concurrence sauvage au sein du transport routier, est destructrice pour les deux secteurs.

\section{Les fondements théoriques du modèle européen d'appel aux forces du marché}

Adossée à la politique de monnaie européenne forte, la politique européenne de la concurrence tranche avec la logique parfois dite keynésienne de l'après guerre. Elle exprime les limites atteintes au début des années 70 par la régulation inflationniste en période d'internationalisation concurrentielle des flux de marchandises et de capitaux. L'objectif est l'adaptation des sociétés européennes à la nouvelle donne mondiale constituée par le passage d'une concurrence pacifiée à une concurrence exacerbée, porteuse de risques et déjà de formes partielles de guerre économique.

\section{Les vertus de la concurrence}

La politique de la concurrence développe le recours à la régulation marchande parce qu'elle pense celle-ci, par principe, comme "bonne" et assimile renforcement de la dynamique concurrentielle et recherche de l'efficacité économique. Si la théorie économique standard a d'abord défini les mérites de la concurrence en l'analysant sur le modèle de la concurrence pure et parfaite, il s'est avéré de plus en plus difficile de transférer les résultats d'un modèle peu robuste, toute imperfection empêchant d'appliquer les enseignements d'un modèle fondé sur la perfection. La justification théorique de la concurrence doit faire appel à d'autres principes d'analyse et se fonde aujourd'hui sur deux grandes constructions :

Hayek propose une justification générale de la concurrence en en faisant un agent de révélation d'information qui permet d'atteindre des objectifs qui ne l'auraient pas été avec d'autres méthodes. En particulier, l'efficacité de la concurrence marchande comme productrice d'information vient de ce qu'elle est seule à pouvoir traiter l'information "tacite", informelle. Le prix synthétise une information en donnant une indication de l'appréciation sociale d'un bien à un moment donné et donc de la combinaison des multiples arbitrages individuels entre acquisition de ce bien, production de ce bien et autres emplois possibles représentés par le coût d'opportunité en monnaie. Ces arbitrages sont éminemment instables, changeants et la plupart du temps inconscients. Les agents économiques ne connaissent donc pas eux mêmes les informations qu'ils vont de fait livrer au marché par leurs comportements. Le mécanisme marchand est pour cela irremplaçable car il ne sera jamais possible de remplacer cette information informelle, inconsciente, par la transmission directe (hors mécanisme marchand) d'une information formelle, codifiée, objectivée, sans en perdre une quantité considérable. A l'inverse, le monopole conduit à une mauvaise utilisation des ressources pour deux raisons principales:

- l'entreprise placée en situation de monopole n'est pas soumise aux mêmes pressions que si elle était soumise à la concurrence d'une multitude de firmes. Elle peut se dispenser de traiter rapidement et correctement l'information et d'y adapter en permanence ses décisions. 
- elle peut transformer en rente son monopole en matière d'obtention et de fourniture d'information. Peu incitée à réduire ses prix, elle va les fixer au-dessus du coût marginal et rien ne garantit que les surprofits ainsi captés soient réintroduits sans gaspillage dans l'économie ${ }^{3}$.

Baumol, Panzar et Willig, avec la théorie des marchés contestables, prétendent montrer que le raisonnement traditionnel néo-classique peut dépasser le cadre des cas extrêmes de concurrence pure et parfaite et justifier un libéralisme qui admette les grandes organisations tout en en refusant une, ... I'Etat. Pour qu'un marché ait un fonctionnement concurrentiel il suffit qu'il soit contestable ; le marché parfaitement contestable peut alors remplacer le marché de concurrence pure et parfaite comme norme et les marchés contestables (imparfaitement contestables) être analysés en référence à ce nouveau modèle norme. La concurrence potentielle a un rôle essentiel, souvent plus important que la concurrence réelle. Dès que le marché est parfaitement contestable, il existe, en fonction de données objectives, techniques (le spectre des technologies à un moment donné et l'état des ressources disponibles), une configuration "naturelle" qui est la configuration efficiente. De plus, le fonctionnement "libre" du marché conduit à s'approcher de cette configuration naturelle. En effet, la concurrence, par son effet de sélection des meilleurs, c-a-d de ceux qui sont les plus rationnels, donc les plus efficients, oblige les configurations réelles à évoluer vers les configurations naturelles efficientes.

Parmi celles-ci prennent place les monopoles naturels, dans des activités présentant des caractéristiques particulières, et notamment, ce qui nous concerne directement, dans beaucoup d'activités de réseau. Dans ces situations en effet des indivisibilités et des économies d'échelle se manifestent fréquemment : un système de réservation informatisé a à peu près le même coût qu'il gère une liaison ferroviaire ou plusieurs. De même existe-t-il de nombreuses économies d'envergure : le production simultanée de services de transport pour les voyageurs et pour les marchandises entraîne une meilleure utilisation des ressources en permettant de combiner les spécificités de l'exploitation (utiliser pour le fret les sillons laissés libres, la nuit, par le trafic voyageurs) et d'utiliser des ressources communes (un système global de sécurité est moins coûteux que la somme de deux systèmes entièrement séparés, l'un pour le fret, l'autre pour le trafic voyageur). Le monopole naturel est efficace et est contraint à l'efficacité, si, au lieu de chercher à le réglementer, les autorités de la concurrence se contentent d'assurer la possibilité de contester le marché en question, c-a-d la liberté d'entrée et de sortie. La démarche s'inscrit logiquement dans les politiques de libéralisation des marchés par la déréglementation avec un nouvel objectif, entretenir la menace de concurrents potentiels afin d'accroître la contestabilité des marchés.

La contestabilité est liée à l'absence de coûts irrécupérables qui dissuaderaient l'entrée en faisant craindre des pertes en capital en cas de besoin de ressortir du marché. Si l'absence de coûts nets d'entrée (ou leur faiblesse) est envisageable dans des secteurs à faible intensité capitalistique et à facteurs de production non spécifiques, il n'en est pas de même dans la plupart des secteurs industriels modernes reposant sur de la production à base technologique complexe et dépourvus des marchés parfaits d'occasion pour les équipements. Les coûts irrécupérables

3 Cette analyse est originale et forte. Elle permet certainement de comprendre l'une des fonctions de la concurrence marchande en économie de production complexe. Elle demande cependant à être confrontée à d'autres analyses du rôle informatif du marché dès lors qu'on veut l'appliquer à la délimitation respective des domaines privé et public. De ce point de vue, deux remarques s'imposent:

- en premier lieu, on peut se demander si le marché synthétise correctement, sans biais, les données dispersées et inconscientes ou si, au contraire, dans la mesure où il rétro-agit sur les perceptions et connaissances, formelles et informelles, des individus, il ne contribue pas à les modeler, ce qui peut conduire à des résultats aberrants ou appauvrissants en termes de fonctionnement. On peut penser à l'analyse keynésienne de la spéculation et à son refus de conférer au marché une quelconque lucidité pour en faire au contraire un lieu d'anticipations conventionnelles et a-rationnelles pouvant entraîner le système dans des cercles vicieux déstabilisateurs. Les risques d'instabilité de fonctionnement peuvent dès lors justifier l'appel à des mécanismes ou procédures non marchandes (et à l'intervention publique) encadrant la production et la transmission d'information.

- en second lieu, il conviendrait de remarquer que le marché mesure difficilement les effets à terme (le marché est "myope"), occulte certaines données économiques et sociales parce qu'elles n'ont pas de traduction marchande et constituent pour lui des sortes d'effets externes (les coûts indirects de l'exclusion des chômeurs du monde du travail comme les effets externes traditionnels). II y aurait alors à se demander si le coût pour l'efficacité sociale de "I'oubli" de ces données, oubli qui contredit le rôle de producteur d'information du marché, est ou non marginal, et sur qui il retombe. 
semblent y être une réalité incontournable et, particulièrement dans les réseaux à lourdes infrastructures. La solution miracle existe cependant !

Systématiquement proposée aujourd'hui, et à l'origine de toute la politique européenne de la concurrence dans les réseaux, elle consiste à confier les coûts irrécupérables à la collectivité. On peut par exemple étatiser ou municipaliser les équipements fixes irréversibles (aéroports, gares, plates formes d'interconnexion,...) afin de diminuer les coûts irrécupérables et créer la concurrence au niveau de leur exploitation (on peut alors songer à la séparation de la gestion de l'infrastructure et de celle de l'exploitation). Les économies d'échelle ou d'envergure sont conservées et gérées en délimitant au plus juste le périmètre du monopole naturel, en le réduisant quand c'est possible par la désintégration des entreprises et la mise en place de nouvelles configurations (socialisation des portions d'infrastructures porteuses de coûts irrécupérables). La libre concurrence doit emplir le reste du domaine et constituer la forme largement dominante de régulation. En outre, la régulation du domaine socialisé doit elle aussi se rapprocher au maximum des règles concurrentielles classiques et de la logique du marché.

\section{L'approche des autorités européennes}

La Commission européenne a repris et consacré l'idée de la séparation de la gestion de l'infrastructure et de celle de l'exploitation. Pour cela il suffit d'organiser l'accès de tous les candidats aux infrastructures. Le monopole ne peut plus se réserver l'exploitation de l'infrastructure mais doit mettre sur le marché les droits d'utilisation, les services d'exploitation constituant alors un marché contestable, livré à la concurrence.. Selon le Commissaire européen à la concurrence, "I'accès des tiers aux réseaux procède d'une logique irréversible", chaque pays restant maître de la forme précise de mise en œuvre du principe au nom de la subsidiarité.

La théorie économique standard a mis en évidence des échecs ou des limites du marché concurrentiel qui justifient traditionnellement des formes de réglementation. La doctrine européenne, en cours de constitution, cherche à en tenir compte, en accompagnant l'extension de la concurrence par des mesures spécifiques, prises au coup par coup, dans le cadre d'un processus par " essais et erreurs ». Nous avons ainsi une politique fondée sur l'extension d'une logique libérale, mâtinée à la marge de règles correctrices ou incitatives. Ainsi veut-elle éviter des entrées opportunistes, certaines firmes se réservant les parties les plus rentables d'un secteur et les autres disparaissant progressivement, laissant non satisfaite une partie de la demande. D'où l'imposition de règles d'accès au réseau, de non discrimination,.. bref, le recours à une concurrence encadrée. Dans le domaine du ferroviaire, les problèmes portent principalement, pour les autorités européennes, sur les risques de concurrence inefficace ou destructrice en matière d'exploitation du fait de comportements opportunistes des firmes et sur la prise en compte des effets externes. En outre, doit être intégrée la fourniture d'un service universel.

Dans le passé, la politique du fer, partait de l'identification entre entreprise publique et service public et découlait de ce fait de la définition du transport ferroviaire comme activité de service public. La transformation de la SNCF en EPIC a introduit une première dissociation entre entreprise publique et service public en attribuant à l'entreprise des activités de service public et des activités commerciales. La politique européenne du fer va plus loin encore en limitant strictement les activités de service public à celles de service universel.

\section{Le modèle européen du service universel}

A la notion traditionnelle de service public tend à se substituer, sous la pression de Bruxelles, la notion de "service universel", défini comme "l'accès à un ensemble minimal de services définis d'une qualité donnée à tous les utilisateurs indépendamment de leur localisation géographique et, à la lumière de conditions spécifiques nationales, à un prix abordable". La fourniture de ce service peut donner lieu à compensation de la part des pouvoirs publics pour la mise en place de tarifs moindres (les tarifs sociaux du chemin de fer constituent l'exemple type).

Le niveau du contenu du service universel est évidemment l'une des grandes équivoques de la notion. Qui appréciera l'étendue du service universel dès lors que celle-ci conditionnera les éventuelles subventions publiques, contrôlées par la Commission et les juridictions européennes de la concurrence?

Le financement des missions de service public se fait actuellement en France, dans le cadre des entreprises publiques, par un système de péréquation qui reste en grande partie peu 
transparent et est de la responsabilité des directions d'entreprise. Bruxelles refuse ce système dit des "subventions croisées" au nom de la vérité des coûts et souhaite isoler les missions susceptibles de compensation, cette compensation donnant lieu à versement public défini et contrôlable par les autorités européennes de surveillance de la concurrence. L'ouverture du marché à la concurrence permet de remettre en cause les formes de péréquation. Des concurrents pourront en effet n'assurer que la fourniture des services les moins coûteux et les proposer à prix inférieur, empêchant la poursuite de surfacturations destinées à compenser les surcoûts des segments plus difficiles. La redistribution entre catégories d'usagers, la redistribution géographique disparaîtront à terme. Les services non rentables soit disparaîtront, soit seront payés plus cher par les clients de façon à devenir rentables, soit seront payés par la collectivité s'ils entrent dans la catégorie du service universel mais après décision publique explicite.

La logique du service public et la logique du service universel n'ont rien de commun. La notion de service universel vient de l'offensive néolibérale américaine et britannique et correspond à la politique Thatcher de substitution au service public britannique d'un système déréglementé, privatisé, marchandisé, incluant le service universel.

On peut les opposer clairement à partir du rapport qu'ils organisent entre le marchand et le non-marchand:

- Le service universel s'inscrit dans une démarche qui donne au marché le rôle essentiel. L'économie est une économie de marché, les biens et services obéissent essentiellement à une logique marchande. II existe des exceptions qui prennent place à l'intérieur de ce contexte général mais la domination générale reste celle du marchand. Le fondement du service universel est distributif et se réfère à la consommation. Les individus doivent pouvoir consommer des services minima dans des conditions permettant à tous d'y accéder. II est donc du domaine de l'assistance. Des individus qui courraient le risque d'être privés du service en fonction de leur pouvoir d'achat doivent avoir la garantie de pouvoir se le procurer dans des conditions décentes. minimum.

On comprend alors que sa logique soit une logique d'exception et de limitation au strict

- Le service public s'inscrit dans une démarche qui donne à la République le rôle essentiel. Celle-ci peut recourir au marché, mais le marché est second et encadré. C'est le non-marchand qui domine le marchand. C'est le politique qui décide en fin de compte. II décide notamment de confier des biens et services au marché et d'autres à une régulation publique qui peut utiliser des éléments marchands mais dont la logique dominante n'est pas celle du marché (parce qu'elle doit atteindre des objectifs que le marché ne peut se donner, indépendance nationale, justice,..). Le fondement n'est plus assistantiel, il renvoie à un mode de gestion de la production, de la circulation et de la répartition des biens et services. Le service public est ce qui, dans la production de biens et services, n'obéit pas à la logique marchande mais, directement, à une logique publique.

On voit alors que l'opposition entre service public et service universel renvoie à la concurrence entre deux ordres susceptibles de gérer le social, l'ordre républicain et l'ordre marchand. Raisonner en termes de service universel c'est promouvoir la logique marchande et vouloir la faire prédominer sur la logique politique, raisonner en termes de service public c'est affirmer la primauté de celle-ci.

\section{L'organisation européenne du ferroviaire}

\section{Un bouleversement de la situation existante}

L'organisation européenne du ferroviaire découle des trois principes de la doctrine européenne : introduire le maximum de concurrence, séparer infrastructures et exploitation, limiter le service public au service universel. On a là un bouleversement complet par rapport à la situation existante.

Dans le modèle des années 60 , la politique du fer, partait de l'identification entre entreprise publique et service public et découlait de ce fait de la définition du transport ferroviaire comme activité de service public. Ce choix se fondait sur des considérations économiques et politiques, 
tenait notamment compte des spécificités du chemin de fer, en décidant que dans le domaine concerné :

- la logique économique était seconde par rapport à une logique politique ; le service public est moyen de la cohésion sociale et assure à tous les membres de la société, en tant que citoyens, des prestations de base indispensables à cette intégration sociale. En particulier, le ferroviaire est moyen de l'aménagement du territoire et créateur de lien social.

- la logique économique ne disparaît pas pour autant mais ne peut s'identifier à une logique marchande ; si le service public intègre des éléments de logique marchande (via le calcul économique public notamment) il a un rôle économique qui dépasse le marché, d'une part en répondant à certaines de ses limites (la prise en compte des effets externes notamment), d'autre part en encadrant le marché (le transport est une activité structurante).

La politique européenne du fer rompt complètement avec ce modèle. Elle limite strictement les activités de service public à celles de service universel faisant de celui-ci un domaine marginal de la vie sociale, une sorte de SMI, Service Minimum d'Insertion, analogue à un RMI. La différence est qu'il porte sur des prestations en nature, des services, et qu'il est concédé à l'ensemble des citoyens mais la philosophie est la même : assurer des conditions minimales de survie dans la société en fournissant des prestations, monétaires ou en nature, minimales. La gestion du fer doit en tenir compte, en particulier via des financements au coup par coup de missions de service universel, mais ne peut se définir sur cette base. La politique globale du fer doit alors s'intégrer à la politique générale de la concurrence et, compte tenu de l'organisation qui existait jusque là, se traduire avant tout par une extension de la concurrence.

La politique de concurrence qui s'appliquera au fer est spécifiée par la considération de la contrainte technologique qu'est le fonctionnement en réseau, fonctionnement qui implique deux choses essentielles:

- une gestion unitaire et intégrée de l'infrastructure ; pour des raisons autant économiques, techniques que de sécurité, il serait dangereux et coûteux de "disperser" l'infrastructure.

- une gestion étatique de la charge d'infrastructure ; les infrastructures constituent des coûts irréversibles importants. Ils empêchent la libre sortie sans coût donc l'entrée et nécessitent d'aménager la contestabilité du secteur en les excluant de la sphère marchande.

On se dirige donc vers un système où des entreprises seront placées en concurrence sur une infrastructure appartenant à l'Etat et gérée par lui. Le rôle de la SNCF doit en être modifié : la direction européenne de la concurrence met en avant la nécessité de lutter « contre la culture de monopole » et d'obliger les firmes, y compris les firmes publiques, à se soumettre au calcul économique. La position dominante dont ont historiquement joui ces entreprises aurait créé une "culture de monopole» qui peut, selon les autorités européennes, conduire, compte tenu des limites du contrôle de l'autorité politique liées à l'asymétrie d'information, à la mise en oeuvre d'une logique d'entreprise, indépendamment des objectifs sociaux qu'ils sont censés poursuivre, aux dépens des usagers (ou «assujettis ") privés de tout recours, voire des contribuables. Le rapport consacré par le Sénat français à la SNCF a largement repris cette idée.

La culture de monopole s'accompagnerait en France de la domination d'une culture technique d'ingénieur alors que l'heure serait à l'intégration d'une culture de marché. Au lieu d'être à l'écoute de la clientèle afin d'adapter l'offre à des demandes changeantes et diversifiées, la culture technique pousse au surinvestissement. Les projets sont mis en avant pour leur apport technologique indépendamment de considérations de rentabilité. La déréglementation, en créant la concurrence d'opérateurs privés, obligerait les grandes firmes publiques à respecter le calcul économique marchand.

Dans ces conditions, la doctrine de la Communauté conduit à l'alignement de l'organisation des chemins de fer sur celle des autres modes de transport:

- l'intégration entre gestion des infrastructures et gestion de l'exploitation doit céder la place à une gestion séparée.

- le transport ferroviaire doit devenir une activité ouverte régulée par la concurrence.

les opérateurs doivent pouvoir être des firmes privées, autonomes, concurrentielles, et mues par la recherche de la rentabilité.

- tout possesseur de capital doit avoir la possibilité de l'investir dans le secteur.

le financement public doit se borner à la subvention d'obligations de service public non rentables imposées aux transporteurs et au financement des infrastructures. 
- tout consommateur de services aura le choix entre plusieurs offreurs en concurrence entre eux, et pourra même organiser lui même son service de transport (transport des entreprises pour compte propre comme c'est le cas dans le transport routier de marchandises).

Séparation gestion de l'infrastructure/gestion de l'exploitation et libre accès au réseau

Elle est organisée par la Directive européenne 91/440 dont l'objectif est de stimuler la concurrence dans l'exploitation du transport ferré. Pour cela, quatre modes d'action sont envisagées :

$1^{\circ}$ ) il convient de garantir aux entreprises ferroviaires un statut d'exploitant indépendant leur permettant de mettre en oeuvre un comportement de marché. Ces entreprises doivent disposer d'un patrimoine, d'un budget et d'une comptabilité séparée. Elles doivent être gérées selon les principes applicables aux entreprises commerciales et donc tendre vers un équilibre financier.

$2^{\circ}$ ) l'efficacité peut s'accroître par une gestion séparée de l'exploitation et de l'infrastructure d'où l'exigence d'établir des comptes distincts et d'organiser une gestion séparée des deux activités. La séparation comptable est obligatoire, elle débouche sur une autonomisation de la gestion qui peut ou non passer par une séparation organique (avec création de divisions distinctes au sein de la même entreprise) ou institutionnelle (création d'entités juridiques indépendantes).

$3^{\circ}$ ) la distinction des activités et l'accroissement de la concurrence et du recours à une logique de marché s'accompagnent du maintien du recours à une logique administrative publique (qui n'implique plus le recours à des entreprises publiques) ; "les États membres gardent la responsabilité générale du développement d'une infrastructure ferroviaire appropriée".

$4^{\circ}$ ) l'ouverture du réseau ; sous des formulations ambiguës et apparemment limitatrices se met en place un processus de libre accès progressif aux infrastructures.

Nous sommes ainsi en présence d'un système à trois niveaux :

1 un responsable du développement de l'infrastructure ; l'Etat payeur

2 un gestionnaire de l'infrastructure, qui peut être une entité publique ou privée. de l'Etat.

3 des gestionnaires de l'exploitation, entreprises ferroviaires autonomes et indépendantes

La logique centrale de gestion de ce système devient une logique marchande. Les comptes, fondés sur la seule mesure des coûts et avantages directement monétisés, doivent être équilibrés et les missions de service public sont pensées comme des exceptions demandant des corrections appropriées. De plus, pour la Direction Générale de la Concurrence, la Directive ne constitue pas un point d'arrivée mais un point de départ dans un processus de restructuration.

L'exemple de la déréglementation du transport aérien montre bien comment les autorités initiatrices surmontent les oppositions en mettant en place un processus limité au départ mais à forte dynamique interne. Les cas des télécommunications, de l'énergie sont venus corroborer cette analyse. A chaque fois nous sommes en présence d'une stratégie gradualiste, visant explicitement à imposer un processus, au besoin malgré les Etats-nations. II en est exactement de même dans le transport ferroviaire. L'application des principes concurrentiels au domaine des réseaux constitue une étape intégrée dans la stratégie de construction du grand marché européen concurrentiel. 


\title{
4 Des dangers de l'approche marchande à l'esquisse d'un modèle alternatif
}

\author{
La séparation des trois niveaux aménagement de l'infrastructure-gestion de \\ l'infrastructure-gestion de l'exploitation
}

L'analyse historique permet de comprendre la logique institutionnelle du développement du chemin de fer comme secteur intégré. L'intégration n'y relève pas d'un processus de concentration comme cela a été le cas dans d'autres domaines mais a pour première raison les conditions d'émergence d'un secteur nouveau : il faut coordonner un ensemble de conditions, développer une stratégie cohérente et le marché ne peut suffire à cette coordination. Par la suite, la gestion intégrée de l'infrastructure et de l'exploitation améliore l'efficacité technique et l'efficacité organisationnelle.

Les arguments relatifs à l'efficacité technique ne jouent plus à partir du moment où les technologies sont stabilisées. L'adaptation réciproque infrastructures/exploitation n'implique plus de choix techniques précis et multiples. En revanche, ils redeviennent d'actualité en cas de progrès technologique. En effet, le transport ferroviaire ne constitue pas seulement un réseau mais aussi un système : les problèmes de sécurité ont bien montré que celle-ci résultait d'un ensemble fonctionnant en système et que l'on ne pouvait traiter un élément indépendamment de son insertion dans ce système ${ }^{4}$. En outre, il est vraisemblable que les nouvelles technologies du rail développent de plus en plus de synergies entre l'infrastructure et l'exploitation.

Les arguments relatifs à l'efficacité organisationnelle ne doivent pas être oubliés. Le niveau d'intégration au sein du système de transport joue sur les coûts d'organisation des relations entre ses éléments, et notamment sur les coûts de transaction. Les sociétés d'exploitation peuvent développer, par rapport à l'Etat, des politiques de chantage au péage, menaçant de renoncer à l'exploitation si le péage n'est pas réduit. Dans ce cas la couverture des investissements effectués ne se fait plus, les infrastructures sont inutilisées et l'Etat est perdant. Le problème se posera surtout en cas de mutation du système technique du transport, mutation qui n'est pas illusoire si des politiques du fret sont mises en place (trains pendulaires, développement d'un fret à grande vitesse, développement du transport combiné,...). Le marchandage autour des contributions de l'infrastructure et de l'exploitation peut être coûteux et entraîner des comportements opportunistes 5 .

\section{L'exploitation concurrentielle}

Divers arguments sont avancés pour justifier la mise en concurrence de l'exploitation :

- la mise en concurrence évite que se constituent des rentes de monopole et instaure une pression à la compétitivité des prix et des services. Dans le cas de la SNCF certains mettent en cause les "privilèges" du statut du cheminot. Plusieurs observations doivent cependant être avancées sur ce point.

La première est que la SNCF n'est pas un véritable monopole en ce qu'elle est soumise à la concurrence de l'avion et à celle, redoutable, de la route. Le résultat est que l'entreprise, loin d'engranger des surprofits, accumule plutôt des pertes. On pourrait rétorquer à cela que les pertes représentent précisément la contrepartie de rentes de situation du personnel. II est possible que l'entreprise joue effectivement de son opacité pour maintenir des avantages divers, voire ait organisé une certaine opacité pour y arriver. II conviendrait alors de régler le problème en

\footnotetext{
4 Pour les TGV l'essentiel du système de signalisation est embarqué, de sorte qu'il doit y avoir cohérence étroite entre matériel et infrastructure, rail et mobile, pour leur conception et leur fabrication mais aussi pour déterminer quotidiennement les conditions optimales de leur utilisation.

5 La répartition des charges en période normale peut-être source de conflits entre administration responsable des infrastructures, gestionnaire de l'infrastructure et sociétés d'exploitation. En cas de modernisation (changement du système de sécurité, du type de rails,..) est-ce de la gestion de l'infrastructure et du coût d'entretien ou est-ce de l'investissement, du développement de l'infrastructure ? Ou établir la différence?
} 
développant la transparence de l'entreprise, en affinant le calcul de ses coûts, en améliorant les procédures de contrôle public. La mise en concurrence est-elle indispensable pour cela ?

La seconde observation est qu'il y a lieu de discuter de ce qu'on nomme "avantages indus" . C'est tout le problème du statut du cheminot d'une part, de la garantie d'emploi de l'autre. Compte tenu des évolutions technologiques, le statut du cheminot est-il aujourd'hui dépassé ? Peut-être, mais ne convient-il pas alors de poser directement le problème et d'engager des négociations sur ce point, plutôt que de confier à la concurrence marchande le soin d'en fixer les contours? L'exemple du transport routier est significatif : la concurrence sauvage qui y règne a modelé un statut social du routier aberrant et la solution ne peut passer que par la réglementation, y compris contre les tendances endogènes du marché qui contribuent à aggraver en permanence les conditions de travail dans la profession.

- dans le même ordre d'idées, et d'un point de vue dynamique, la concurrence obligerait les entreprises de chemin de fer à faire la chasse aux gaspillages, à innover, à s'adapter rapidement aux évolutions du marché. L'absence de concurrence sur le marché précis du transport ferroviaire, joint à la lourdeur de l'organisation bureaucratique traditionnelle des entreprises ferroviaires, a sans doute freiné la prise en compte des mutations de la demande de transport. Faut-il absolument une concurrence effective pour cela, sachant que cette concurrence a, par ailleurs, de multiples inconvénients ? Une autre solution ne serait-elle pas à rechercher dans la transparence de l'entreprise, sa démocratisation pour la mettre davantage au service du public et lui faire remplir mieux ses fonctions de service public, fonctions qui ne se limitent pas à satisfaire les seules demandes exprimées par le marché.

- les arguments relatifs à l'efficacité de la libre concurrence sont de peu de portée. L'expérience de l'ouverture de la concurrence montre qu'on aboutit, non à une libre concurrence, mais à une situation d'oligopole différencié, chaque entreprise se spécialisant dans un sousmarché particulier, dans le cas britannique une zone géographique. Sur ce sous-marché, chaque firme exploite son avantage particulier comme monopole. La généralisation du yield management illustre d'ailleurs ce comportement, qualitativement distinct de celui de concurrence pure et parfaite censé conduire à l'optimum. En outre, la possibilité d'aboutir à une parfaite contestation des marchés de transport ferroviaire est douteuse. Les coûts irrécupérables ne se limitent en effet pas aux coûts d'infrastructure pris en charge par l'Etat. Un entrant doit opérer un investissement commercial qui, si l'entrée tourne mal, se révèle définitivement perdu. L'exemple du transport aérien montre qu'au bout d'un certain temps l'entrée se limite à des repartages entre grandes compagnies, seules à même de soutenir les investissements nécessaires en systèmes d'information et en politique commerciale.

Il faut ajouter à cela les limites de la concurrence :

- La première limite est la possibilité qu'elle dégénère en situation de concurrence destructrice. La déréglementation du transport aérien nous a ainsi appris que les coûts systémiques de la concurrence (prix de prédation et guerres de prix, entrées opportunistes,..) pouvaient être élevés. Les caractéristiques du ferroviaire et notamment le poids des immobilisations (en infrastructures mais aussi en frais fixes d'exploitation) conduisant à une très forte différence entre coût moyen et coût marginal renforcent ces risques 6 .

- La deuxième limite concerne les effets externes. Leur prise en compte, dans le cadre de la concurrence intermodale, est nécessaire mais il est plus facile d'en tenir compte quand les

6 Les entreprises ferroviaires (et le cas du fret est significatif), avec des coûts marginaux décroissants et surtout des coûts marginaux très largement inférieurs aux coûts moyens, sont potentiellement confrontées à des acheteurs qui peuvent minimiser leur dépense en achetant au coût marginal. Le raisonnement standard de la théorie économique montre que s'ils offrent un peu plus que le coût marginal, l'offreur, qui subit de toutes façons les frais fixes, à intérêt à leur vendre.

Le développement de la concurrence, sous la forme de la concurrence intermodale, allait déjà dans ce sens comme le montre l'exemple du transport aérien. La concurrence des autres moyens de transport (concurrence avion/rail/route) a rendu la péréquation entre lignes rentables et non rentables difficile à tenir. II faut en effet diminuer les prix sur les lignes bénéficiaires pour résister à la concurrence donc limiter les provisions qui permettaient de refinancer les lignes déficitaires.

Pour une analyse plus détaillée, nous nous permettons de renvoyer à « Les conséquences de la séparation comptable entre gestion de l'infrastructure et gestion de l'exploitation ferroviaires », Rapport pour le Comité Central d'Entreprise de la SNCF, par C.Barrère, E.Bibault, D.Debatisse, M.N.Jubenot. 403 pages. Ceras. Université de Reims. Juillet 1996. 
pouvoirs publics ont à traiter avec un monopole réglementé que lorsqu'ils ont à le faire avec une pluralité d'exploitants

- La troisième est l'existence d'une situation de monopole naturel. II convient donc de se demander si l'exploitation ferroviaire participe ou non du monopole naturel, alors que la politique européenne l'admet implicitement ou explicitement pour les infrastructures ferroviaires. S'il existait des économies d'échelle (plus le volume de service augmente, plus son coût moyen diminue) en matière d'exploitation, il conviendrait d'avoir un exploitant unique. S'il existait des économies d'envergure (il est moins coûteux d'avoir une seule firme produisant les biens $A$ et $B$ que deux firmes, chacune ne produisant que l'un des biens), économies liées à la gestion par une même firme des infrastructures et de l'exploitation, il conviendrait d'avoir une entreprise unique, chargée et des infrastructures et de l'exploitation, comme c'était le cas traditionnellement en France. Ces économies d'échelle et d'envergure, auxquelles on doit associer la forme particulière des économies de réseau (il est plus efficace d'avoir une organisation de l'offre et de la demande en réseau que par segments autonomes), portent et sur les coûts de production et sur les coûts d'organisation. La discussion est technique et délicate et les avis d'experts ne permettent pas, dans l'état actuel des connaissances sur le chemin de fer, de se prononcer définitivement sur le niveau et le contenu des économies de réseau, d'échelle et d'envergure qui existent dans le transport ferroviaire. De sérieux arguments montrent cependant qu'il existe de telles économies impliquant qu'il est plus économe :

1 que les infrastructures soient utilisées et par le transport de fret et par celui de voyageurs

2 que l'exploitation concerne en même temps le fret et les voyageurs

3 qu'une seule entreprise gère la totalité du réseau.

\section{Infrastructures, exploitation et service public}

La politique des réseaux a conduit, en Europe, dans tous les cas, à une segmentation en dissociant des demandes en fonction de la disposition à payer qu'elles révèlent. D'où la dissociation entre créneaux rentables et créneaux non rentables. On sait que, dans le cas du chemin de fer, les rentabilités des différents segments du réseau sont fort différentes. L'ouverture à la concurrence signifie alors le développement de la concurrence sur les portions rentables et la tendance à l'abandon des segments non rentables, sauf à redresser leur rentabilité par financement public.

Un pilotage par le marché risque d'aggraver la centralisation parisienne et d'opérer une forte segmentation au sein des services régionaux. En outre, dans le chemin de fer qui fonctionne en système, l'appréciation de la rentabilité individuelle d'une ligne est équivoque. Certaines lignes non rentables peuvent servir en partie à alimenter les lignes rentables, qui, sans elles, ne le seraient plus. En France, l'organisation du trafic pour "rabattre" les voyageurs vers les points d'accès aux TGV est significative de cette logique.

L'on doit également tenir compte du risque lié à l'imperfection de l'information. Le cas d'Eurotunnel est significatif de ces problèmes. Une liaison en fait rentable mais dont la rentabilité n'est pas ex ante assurée parce qu'elle dépend d'éléments aléatoires (et l'on sait que la prévision du recours au ferroviaire est délicate en période de forte concurrence intermodale) peut être délaissée par des exploitants privés averses au risque alors qu'une firme publique, moins contrainte par une rentabilité de court terme, assumerait le risque.

La logique de la segmentation marchande du transport ferroviaire peut être contrebalancée par l'intervention de l'Etat ou des régions pour inciter les exploitants à assurer certaines missions de service public. L'efficacité de ce contrepoids en période de crise des finances publiques est cependant très douteuse et l'est d'autant plus qu'une partie des missions de service public ne peut prendre place dans le cadre restrictif du service universel conçu comme mécanisme redistributif. Le transport régional et local se justifie, non seulement par les services qu'il rend à la population, mais aussi par un bilan supérieur à celui des autres modes de transport en matière d'externalités.

De ce point de vue, la concurrence au sein de l'exploitation, avec le sacrifice des segments les moins rentables, risque de forcer la main à l'Etat aménageur du territoire. II faudra bien en période de crise financière qu'il adapte son offre d'infrastructures à la demande des exploitants, d'où une " adaptation par le petit côté ". Le pilotage des infrastructures par l'exploitation et la demande immédiate risque de se substituer au rôle structurant d'un Etat responsable du devenir social, anticipant sur les besoins de long terme et raisonnant en besoins 
sociaux et non en dispositions à payer. Or, l'analyse moderne de la croissance telle que la conduit la théorie de la croissance endogène met l'accent sur le rôle de l'État dans la production d'externalités positives, notamment par la production d'infrastructures, alors que la logique marchande tend à sous-investir en capital privé et en capital public et, paradoxalement, à freiner la rentabilité du capital privé car celle-ci profite des équipements publics.

La directive européenne qui concerne le chemin de fer définit comme premier objectif l'accroissement de l'intégration de l'ensemble des modes de transport. Or, curieusement, cette intégration commence par la désintégration du ferroviaire (séparation infrastructure/exploitation et ouverture du réseau) pour aboutir à une intégration par le marché. L'intégration renvoie à deux réalités : l'intégration du ferroviaire de façon à prendre en compte la réalité d'un système ferroviaire, l'intégration de l'ensemble des modes de transport de façon à prendre en compte la réalité d'un système global de transport.

L'intégration du ferroviaire était de la responsabilité de l'entreprise publique et se faisait, via une régulation administrée, par des politiques du rail qui découlaient de schémas nationaux d'activité, d'une centralisation des recettes et de leur réallocation selon les besoins. La désintégration opérée casse ce mécanisme, interdit explicitement les péréquations et redistributions, et propose une réintégration par le marché. La coordination marchande permetelle d'assurer mieux que la coordination administrative ou institutionnelle l'intégration en réseau et le fonctionnement efficace de ces réseaux ? Si le marché réintègre il opère en même temps une sélection, biaisée par la seule considération des coûts et avantages immédiats enregistrés par le marché. La correction par des opérations ponctuelles de financement de missions de service public ne peut suffire. C'est pourquoi le recours à la régulation marchande et à l'intégration marchande du ferroviaire doit prendre place dans un cadre qui oriente, conditionne et corrige cette régulation et qui est celui d'une politique publique explicite du rail.

L'intégration des modes de transport, par le marché, demande elle aussi à être précisée. Est-ce le choix pour toute relation entre différents modes et la consécration du moins coûteux, avec combinaison par tronçons, selon le principe d'économie ? S'il est clair que le service public de transport ne s'identifie pas avec un service public dans lequel tout passerait par le rail, encore convient-il d'aboutir à des comparaisons de coûts et d'avantages cohérentes et réalistes. Le critère d'économie et de rendement est-il privé ou social ? et que penser alors de la non prise en compte des effets externes?

L'intégration peut être d'autant moins confiée au seul marché qu'il faudrait coordonner des décisions marchandes et des décisions non marchandes, liées aux politiques du rail, de la route, de l'eau, de l'avion, avec une multitude de niveaux décisionnels différents (Union Européenne, États, régions, départements, communes, entreprises). Si les effets externes sont peu importants par rapport aux effets marchands directs, cela n'est pas grave. Leur non prise en compte ne fausse le calcul économique qu'à la marge. Si, au contraire, les effets externes sont importants, voire plus importants que les effets directs, le calcul marchand n'a plus de sens.

Pour une nouvelle articulation marchand/non marchand et public/privé

L'analyse ci-dessus conduit à livrer au débat quelques propositions analytiques que l'on peut récapituler comme suit :

Les problèmes du transport ferroviaire sont des problèmes d'avant-garde à condition de ne pas les prendre " par le petit bout de la lorgnette ", en ne s'intéressant qu'au déficit financier. Ils concernent l'articulation entre service public et service commercial donc entre jeu du marché et limites du marché, l'articulation entre régulation marchande et régulation non marchande. Ils se posent et se poseront de plus en plus à l'ensemble des secteurs et des entreprises. L'économie aujourd'hui a besoin de calcul marchand et d'incitations marchandes mais aussi de calcul non marchand et d'incitations non marchandes : il faut être compétitif mais en même temps créer des emplois, il faut réduire les coûts mais en même temps réduire la pollution,... Le problème de l'articulation entre marchand et non marchand est le problème économique du XXI ${ }^{\circ}$ siècle.

Le modèle bruxellois proposé pour répondre à la crise du modèle de service public des années 60 , dans le contexte d'une progression et d'une intensification de la logique marchande, est fondé sur l'hégémonie d'une régulation marchande, n'admettant que quelques exceptions 
marginales, cas particuliers et strictement circonscrits. Alors que le problème est d'articuler logique marchande et logique non marchande, il transforme le service public, via la notion de service universel, en exception. II est incapable de résoudre les problèmes du transport parce que le marché connaît des limites décisives pour gérer un secteur de ce type, marqué par les caractéristiques de ses immobilisations. II risque d'entraîner une segmentation de l'offre ferroviaire, quelques créneaux rentables étant livrés à la concurrence et des zones d'ombre et de dégradation régnant sur les segments non rentables.

La solution aux problèmes du ferroviaire ne peut consister en le maintien du statu quo. La réflexion sur l'adaptation du service public à une société en crise et en profonde mutation n'a pas connu le développement nécessaire, ce qui n'est pas sans effets sur la crise du secteur public et le laisse quelque peu désarmé devant l'extension de la logique marchande. La solution à la crise du rail passe par la définition d'une politique européenne des transports fondée sur deux principes:

- il n'y a pas de solution hors d'une nouvelle articulation entre régulation marchande et régulation non marchande. La séparation entre marchand et non marchand n'est pas une séparation topologique entre un domaine qui serait celui du marché et un autre qui serait celui de l'Etat comme on l'avait longtemps conçu. II y a partout et du marchand et du non marchand. II y a toujours une articulation entre réglementation et marché. En outre, le marché n'a pas de logique univoque, tout dépend de ce qui est à côté de lui ou de ce qui l'encadre. La lutte contre la concurrence sauvage, particulièrement présente dans le domaine du transport, est indispensable pour tirer du fonctionnement marchand des effets bénéfiques et non destructeurs. Cela passe par la primauté d'une logique de service public qui utilise la logique marchande mais l'encadre et l'oriente. La forme, publique ou privée, des organismes gérant le transport est alors secondaire par rapport au contenu de la politique globale du transport qui s'impose à eux.

- il n'y a pas de solution aux problèmes des entreprises ferroviaires hors d'une politique globale des transports, plurimodale et incluant opérateurs mais aussi utilisateurs du transport (en premier lieu les chargeurs pour le fret). Réformer le ferroviaire sans toucher à la concurrence sauvage qui sévit dans le fret routier ou à l'anarchie de la création d'infrastructures routières est illusoire.

\section{Bibliographie}

en français :

Barrère $(C)$, Bibault $(E)$, Debatisse $(D)$, Jubenot (MN), "Les conséquences de la séparation comptable entre gestion de l'infrastructure et gestion de l'exploitation ferroviaires", Rapport pour le Comité Central d’Entreprise de la SNCF, Paris, 1996.

Boiteux (M), "Concurrence, régulation, service public. Variations autour du cas de l'électricité", Futuribles, n²05, janvier 1996

Cartelier (L), Fournier (J), Monnier (L) Critique de la raison communautaire : utilité publique et concurrence dans I'Union Européenne, Economica, Paris, 1996

Commissariat Général du Plan, La régulation des services publics : concilier efficacité et équité, Eska, 1996

Commissariat Général du Plan, Transports : pour une cohérence stratégique, CGP, Paris, 1993

Commissariat Général du Plan, Les réseaux de services publics dans le monde. Organisation, régulation, concurrence, sous la direction de M.Walgrave, Aspe Europe éditions, Paris, 1995

Conférence européenne des ministres des transports, La privatisation des chemins de fer, service des publications de I'OCDE, Paris, 1993 
Conférence européenne des ministres des transports, Infrastructures et systèmes de transport pour une nouvelle Europe, service des publications de I'OCDE, Paris, 1994

Curien (N) et Dognin (E), "Le service universel : quelle valeur ?, quel coût ?, quel financement ?", Annales des télécommunications, 50, n².

Forum Alternatives Européennes, France, Europe, quel avenir pour le rail ?, FAE, Paris, 1996

Fournier (J), Le train, I'Europe et le service public, Editions Odile Jacob, Paris, 1992

Le Duc (M), Services publics de réseau et Europe, Les exemples de l'Allemagne, de I'Espagne, de I'Italie et du Royaume Uni. Les études de la documentation française, Paris, 1995

Stoffaës (C) Services publics, Questions d'avenir, Editions Odile Jacob-CGP, Paris, 1995 en langue anglaise :

Button (K.J) and Pitfield (D) (eds), Transportation Deregulation, an International Movement, Macmillan, Basingstoke, 1991

Verhoef (E), The Economics of regulating Road Transport, Edward Elgar, Cheltenham, 1996 